\title{
SIGNOS NOTARIALES MEDIEVALES DE LA COLECCION DE PERGAMINOS DEL ARCHIVO HISTORICO MUNICIPAL DE ELCHE
}

\author{
Ana María Navarro Escolano
}

Uno de los elementos que destaca al observar un documento privado, es un signo que va al final del mismo. Se trata del "signum" notarial. El papel que desempeña en la diplomática privada es fundamental, ya que se trata de uno de los factores de validación, pues con su aposición se garantiza la validez de esta carta. (1)

Rolandino (2) lo define como uun sello manuscrito de puño y letra del notario». Según éste, su origen fue un anagrama arbitrario, un enlace de letras con la cruz y esto hace que a veces el signo notarial sirva de crismón.

Valls Subirá (3) opina que su nacimiento se vinculará a una cruz trazada por el notario. Tras la lectura del texto y la aquiescencia de los ineresados, en tres de los ángulos de aquella - el notarioharía un punto; el cuarto lo marcaría el otorgante. Este signo tan

(1) A. Giry, «Manuel de Diplomatique» New York, p. 616 y sig.

(2) R. Nuñez Lagos, «El documento medieval y Rolandino Madrid, 1915, p. 162.

(3) C. Valls Subirá, $* E l$ 'signum' notarialu, en Centenario de la ley del notariado. V. II, Tomo 2 (Barcelona 1962), p. 9 
sencillo se irá complicando según el gusto y la personalidad de cada notario, ya que el signo es también un elemento de identificación de estos, y cada uno tenía el suyo propio (4).

El poseedor de estos signos, el notario, necesitaba pasar por unos exámenes y por la designación - por parte del rey o del señor - para ejercer este trabajo (5). En el ámbito de la Corona de Aragón y a través de la obra de Bono (6), vemos que los notarios de Aragón sólo podían ejercer en aquella población en la que eran autorizados. Valls Subirá (7) ofrece el nombramiento de un notario que actuará en Cataluña (8). Los Fori Antiqui (9) recogen carias rúbricas dedicadas a los notarios, en una de ellas se indica que los notarios de la ciudad de Valencia podrán actuar por todo el Reino (10).

No es nuestra intención el estudio de la evolución de la institución notarial, y para ello remitimos al trabajo bibliográfico de Trenchs (11) y a la obra de Bono (12).

El presente trabajo pretende ofrecer una relación de notarios con sus correspondientes signos notariales, pero obtenidos de los Pergaminos del Archivo Histórico Municipal de Elche. Se han extraido de las colecciones facticias realizadas por Pedro Ibarra y que en el inventario del Archivo reciben el nombre de "Páginas de Oro" y «Pergaminos Notables» (13). La finalidad del estudio al principio puede parecer banal, pero hemos de tener en cuenta que de día en día van adquiriendo mayor transcendencia los estudios sobre protocolos notariales (14) y, puesto que el signo es un elemento identificador del notario, puede servir para completar series de un notario determinado, su campo de trabajo, etc.

(4) C. Valls Subirá, «El 'signum'... p. 21

(5) M. Carcel Orti, «Un formulario notarial del siglo XVII de la Real Audiencia de Valenciav. Saitabi, XXIX 1979, p. 83.

(6) J. Bono, Historia del Deiecho Notarialu Madrid 1979.

(7) C. Valls Subirá, «El 'signum'... p. 13.

(8) Vid. Nota 7.

(9) M. Dualde Serrano, „Fori Antiqui Valentiae», Madrid-Valencia 1950-67 p. 286 y sig.

(10) M. Dualde Serrano, «Fori Antiqui...» p. 287.

(11) J. Trenchs, «Bibliografía del notariado en España siglo $X X$. En estudios históricos y documentos de los Archivos de Protocolosw IV 1974, p. 193-230.

(12) J. Bono, "Historia del Derecho....

(13) A. Ramos Folqués, *Inventario del Archivo Municipal de Elchew 1974 p. 25-28 Perga minos Notables Páginas de Oro, p. 17-23.

(14) A. Eiras Roel y Colaboradores, «La historia social de Galicia en sus fuentes de protocolosw. Santiago 1981 . 
Los signos pertenecientes a los pergaminos del Archivo Histórico Municipal de Elche, corresponden a los siglos XIII, XIV y XV, siendo el más antiguo el de 1258 y el mâs moderno - de los aquí recogidos - de 1483. En total se recogen los signos de 65 notarios, de ellos cinco pertenecen a notarios del siglo XIII, cuarenta y uno al siglo XIV y veinte del XV.

Además de los signos, debajo de los cuales figura un número orden, y el nombre del notario, presentamos unos cuadros donde los notarios se recogen por orden alfabétuco, al igual que el lugar donde pueden ejercer, la fecha en que los documentamos y el número de orden. Digamos finalmente que junto a los signos se ha recogido la palabra «signum» o «senyal».

Nombre del Notario Lugar donde ejerce Año $\begin{aligned} & N^{\circ} \text { de Fondo } \\ & \text { orden }\end{aligned}$

Andreu Fira

Anthoni Arrufat

Anthoni Auret

Anthoni Fire

Anthoni Granyana

Apparici Gonçalvez

Berengari Sarta

Bernardus de Avarsone

Bernardus Franqueti

Bernardus de Jonquerio

Bernardus Textoris

Bernardus de Vellacho

Bernat Tavila

Bertrandus Camuntada

Didacus Gartie

Egidius Petri de Buysan

Ferrando Martines

Francesc Ballester

Francesch de Encalla

Francesch de Mealla

Franciscus Bataller

Guillelmus Çatorre

Guillelmus de Ginestost

Guillelmus de Solanis

Iacobus Felemir

R. Aragón y Valencia
Valencia
P.t.l.t. R. A. (16)
Elche
P.t.l.t. R. A.
Orihuela
Real
Real
Real
Real

1448

1319

1384

R. de Aragón

Valencia

Guardamar

Real

Real

1382-1437

1435-1455

1443

1385

1306

1340

1393

1340

1316

1323

1358

1325

Real

1478

Elche y Crevill.

1337

Orihuela

Elche

Elche

Valencia

R. Aragón

Orihuela

Real

Valencia

1307

1321

1384

1483

1316

1807

1296

1383

$\begin{aligned} 58 & \text { P.N. (15) } \\ 16 & \text { P.N. } \\ 39 & \text { P.O. (17) } \\ 35 & \text { P.N. } \\ 51 & \text { P.N. } \\ 55 & \text { P.O. } \\ 41 & \text { P.O. } \\ 7 & \text { P.O. } \\ 27 & \text { P.O. } \\ 43 & \text { P.O. } \\ 26 & \text { P.O. } \\ 14 & \text { P.O. } \\ 19 & \text { P.O. } \\ 29 & \text { P.O. } \\ 21 & \text { P.O. } \\ 61 & \text { P.O. } \\ 25 & \text { P.O. } \\ 10 & \text { P.O. } \\ 18 & \text { P.O. } \\ 40 & \text { P.0. } \\ 65 & \text { P.N. } \\ 13 & \text { P.O. } \\ 8 & \text { P.O. } \\ 4 & \text { P.O. } \\ 36 & \text { P.N. } \\ & \end{aligned}$

(15) P.N. (Privilegios Notables.)

(16) P.t.l.t. R.A.(Para toda la tierra del Reino de Aragón.)

(17) P.O. (Páginas de Oro.) 
Iacobus Fire

Iacobus Mirons

Iohannes de Sancto Felice

Jacme Trilles

Jacme Julian

Joan Raols

Johan de Castell

Johan Cornella

Johan Gomiz

Johan Martinez de Peralta

Johan de Malla

Johan de Quexans

Juan Ponz

Laurentis Moratori

Lorenç Pinyol

Ludovicus Carcasona

Miguel de Lorens

Miguel Martinez del Moral

Martinus de Boyl

Nicolai Petri

Nicolau Yuanyes

Pere Bataller

Pere Codines

Pere Cornella

Pere Cornella

Pere Dolo

Pere Julian

Pere Sanchez de Llorqua

Pere Talsa

Perot Marchesi

Petrus Batallerii

Petrus Camanyas

Petrus Granyana

Petrus Lupen

Petrus Luppen

Petrus de Podio Iohannia

Petrus Vital

Raymundus Bajulis

Raimundus Vitalis

Raimundus Coventi

Elche
R. de Valencia
Valencia
R. de Aragón
Elche
Alicante
Elche
Elche
Elche
R. de Aragón
P.t.l.t. R. A.

Orihuela y Elche

Teruel

Valencia

Elche

Valencia

Teruel

Teruel

Real

Orihuela

R. de Valencia

Real

P.t.l.t. R. A.

Elche

Elche

R. de Aragón

Elche

Elche

Orihuela

Real

Elche

Real

P.t.l.t. R.A.

Real

Real

R. de Aragón

Real

Real

R. de Aragón

Valencia orden

$1316-1368$
1340
1478
1368
1312
1304

1384

1424

1442

1394

1452

1439-1444

1332

1316

1384-1395

1478

1258

1258

1381

1443

1335

1438

1447

1395

1426

1335

1310

1425

1307

1280

1388-1394

1481

1461

1269

1324

1378

1367

1420

1399

1321
31 P.N.

28 P.O.

62 P.O.

32 P.O.

12 P.O.

6 P.O.

$37 \quad$ P.O.

48 P.N.

54 P.N.

45 P.O.

59 P.N.

53 P.N.

22 P.O.

15 P.O.

38 P.o.

63 P.O.

2 P.O.

1 P.O.

34 P.N.

56 P.O.

23 P.O.

52 P.N.

57 P.N.

44 P.O.

50 P.N.

24 P.O.

11 P.N.

49 P.N.

9 P.O.

3 P.O.

42 P.O.

64 P.O.

60 P.N.

5 P.O.

20 P.O.

33 P.O.

30 P.O.

47 P.O.

46 P.O.

17 P.O. 


\section{SIGLO XIII}

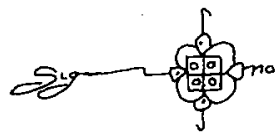

MIGUEL MARTINEZ DEL MORAL

(1)

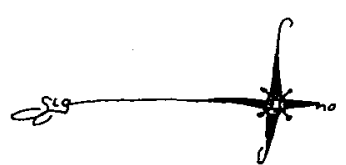

MIGUEL DE LORENS

(2)

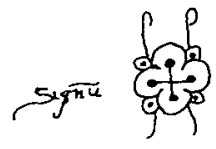

PEROT MARCHESI

(3)

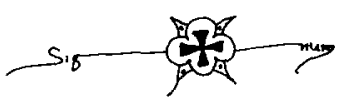

GUILLELMUS DE SOLANIS

(4)
एंडिकर क्षि

PETRUS LUPEN

(5) 


\section{SIGLO XIV}

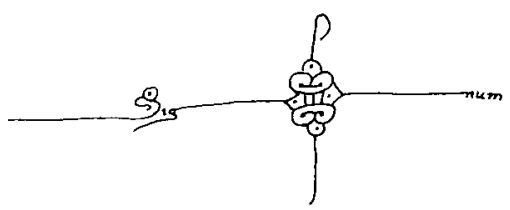

JOAN RAOLS

(6)

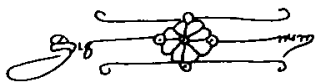

GULLELMUS DE GINESTOST

(8)

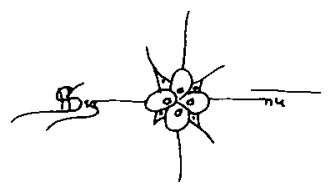

FRANCESC BALLESTER

$(10)$

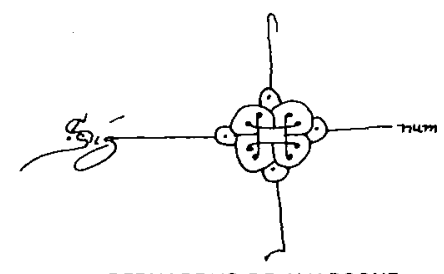

BERNARDUS DE AVARSONE

(7)

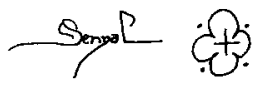

PERE TALSA

(9)

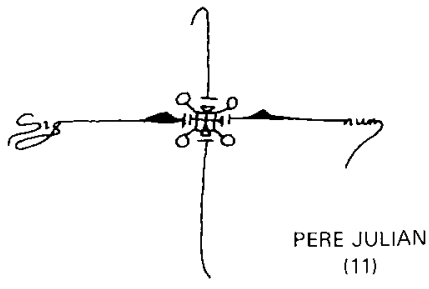




\section{SIGLO XIV}
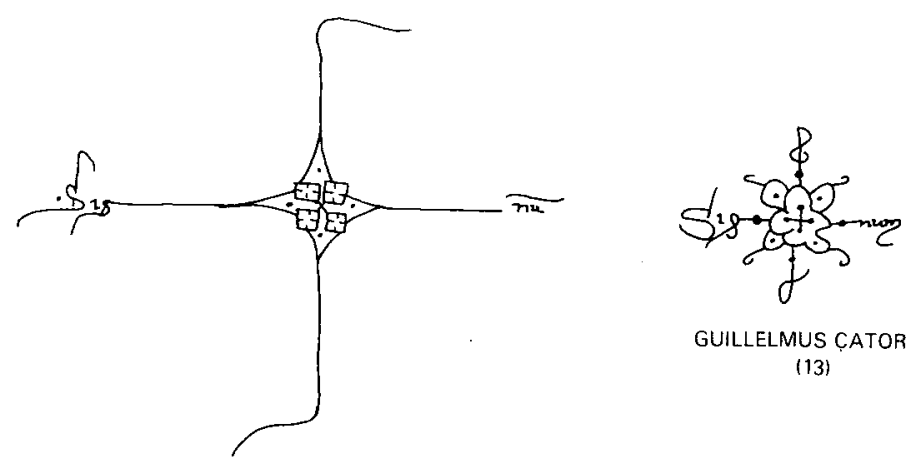

GUILLELMUS CATORRE

(13)

JACME JULIAN

(12)
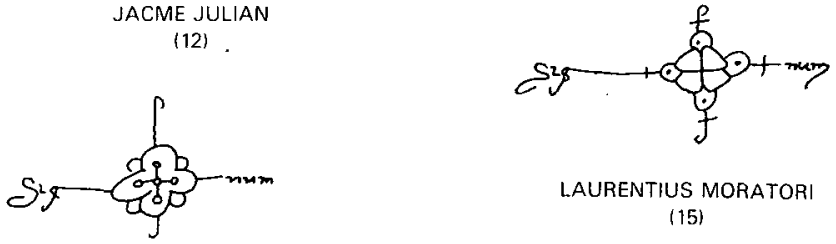

LAURENTIUS MORATORI

(15)

BERNARDUS DE VELLACHO

(14)

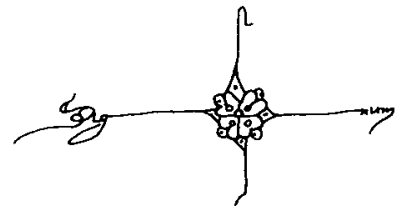

RAIMUNDUS CONVENTI

(17)

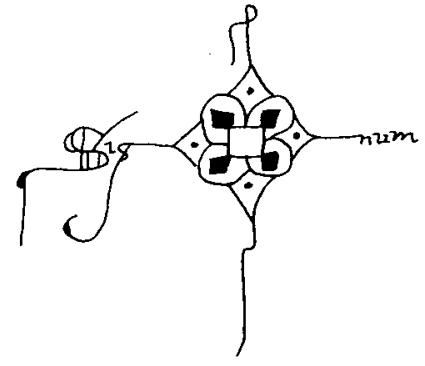

ANTHONI ARRUFAT

(16) 


\section{SIGLO XIV}

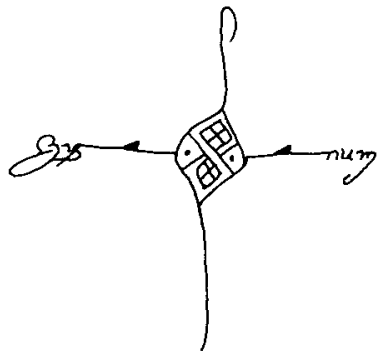

FRANCESCH DE ENCALLA

(18)

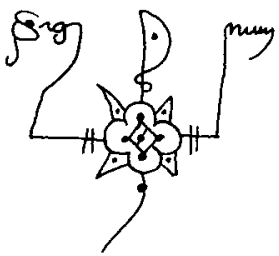

DIDACUS GARTIE

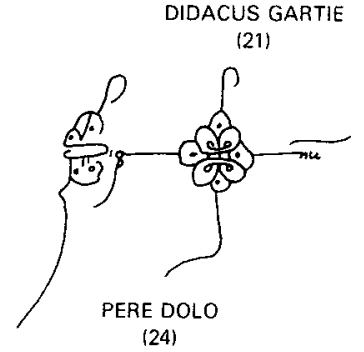

(24)

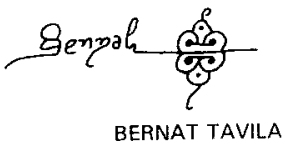

(19)

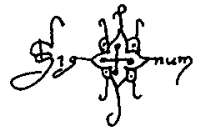

PETRUS LUPPEN

(20)

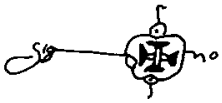

JUAN PONZ

(22)

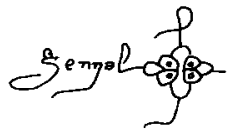

NICOLAU YUANYES

(23)

FERRANDO MARTINES

(25) 


\section{SIGLO XIV}

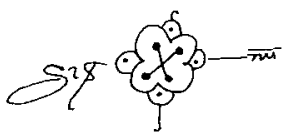

BERNARDUS TEXTORIS

(26)

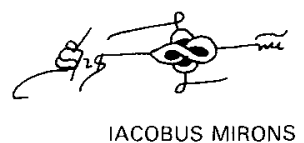

(28)

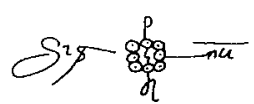

BERNARDUS FRANQUETI (27)

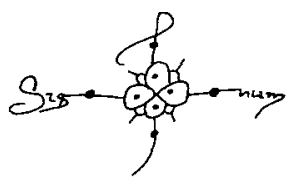

BERTRANDUS CAMUNTADA

(29)

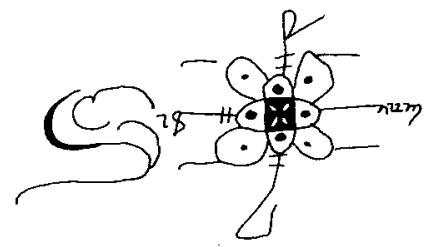

PETRUS VITAL

(30)
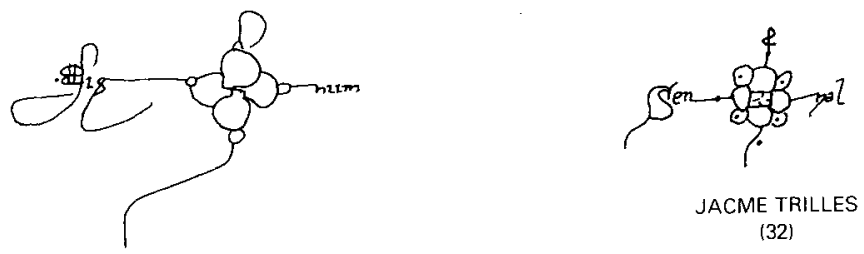

IACOBUS FIRE

(31) 


\section{SIGLO XIV}

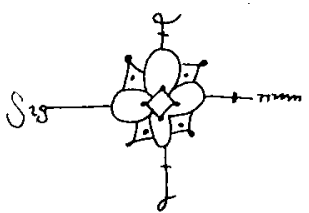

PETRUS DE PODIO JOHANNIS

(33)

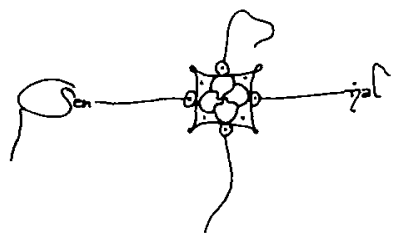

ANTHONI FIRE

(35)

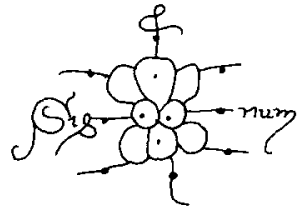

MARTINUS DE BOYL

(34)

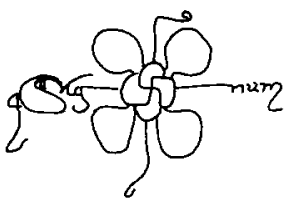

IACOBUS FELEMIR

(36)

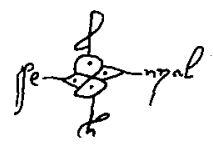

JOHAN DE CASTELL

(37)

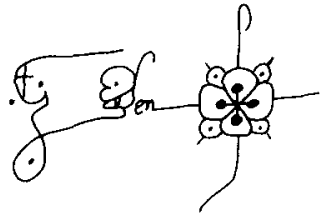

LORENC PINYOL

(38)

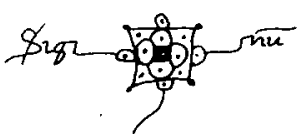

ANTHONI AURET

(39)

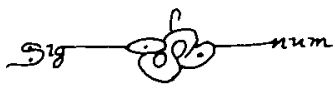

FRANCESCH DE MEALLA

(40) 


\section{SIGLO XIV}

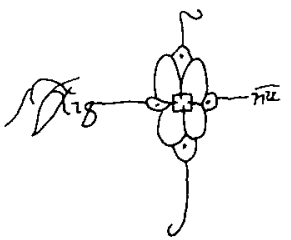

BERENGARI SARTA

(41)

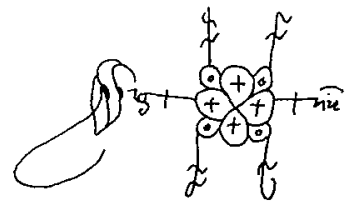

BERNARDUS DE JONQUERIO

(43)

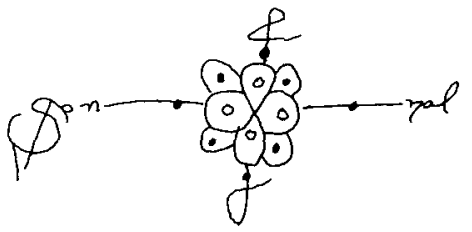

JOHAN MARTINEZ DE PERALTA

(45)

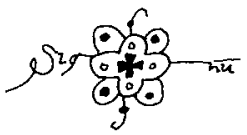

PETRUS BATALLERI!

(42)

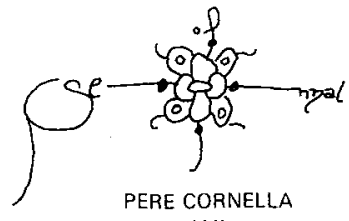

(44)

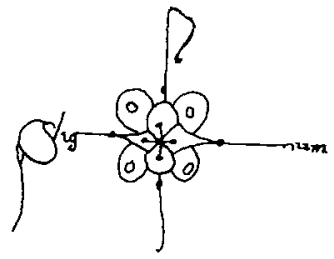

RAIMUNDUS VITALIS

(46) 


\section{SIGLO XV}

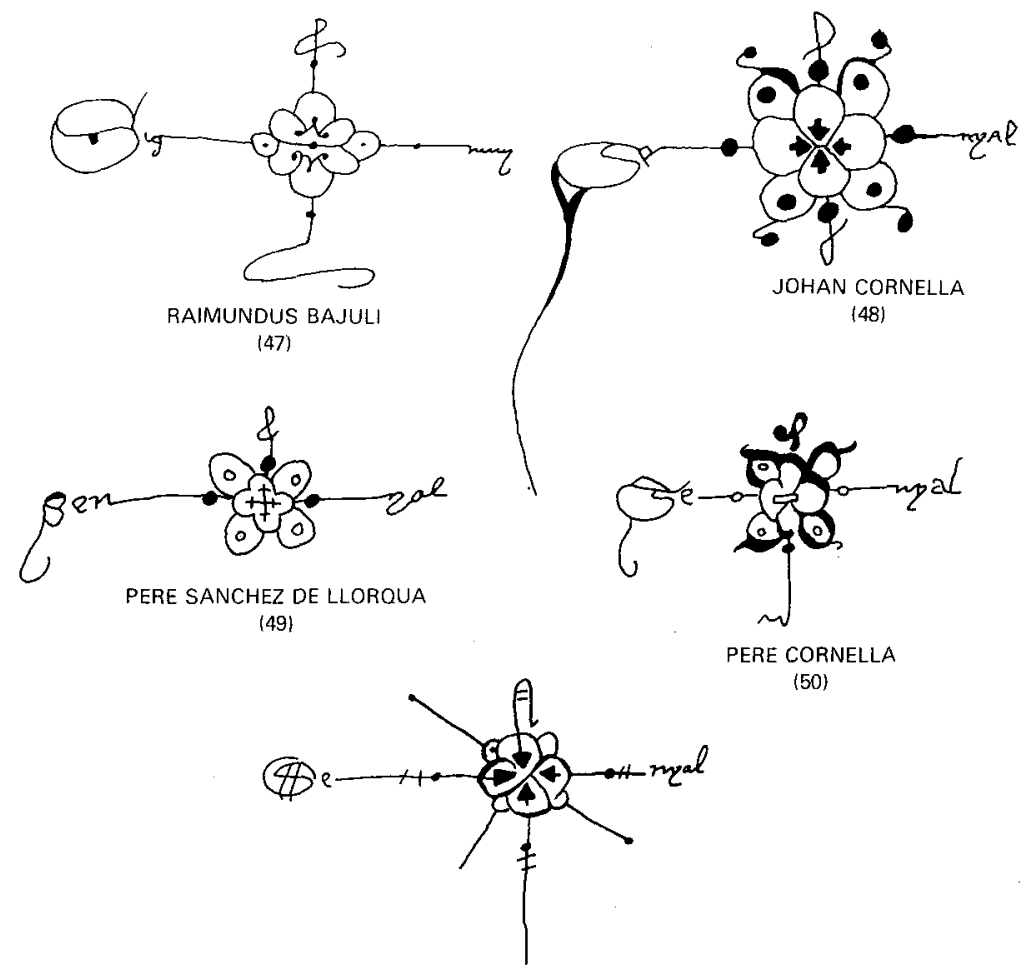

ANTHONI GRANYANA

\{51\}

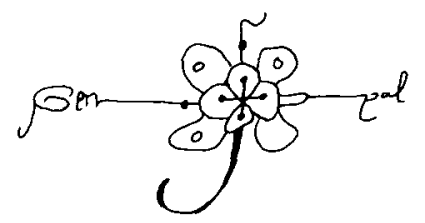

PERE BATALLER

(52)

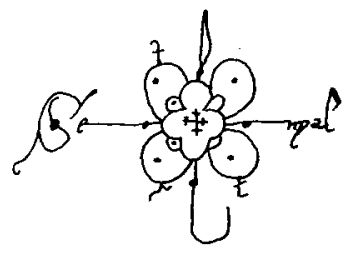

JOHAN DE QUEXANS

(53) 


\section{SIGLO XV}

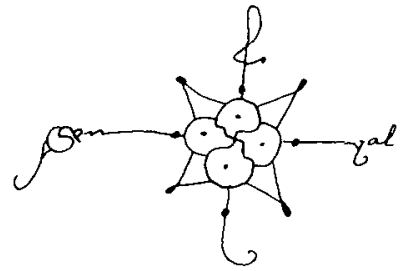

JOHAN GOMIZ

(54)

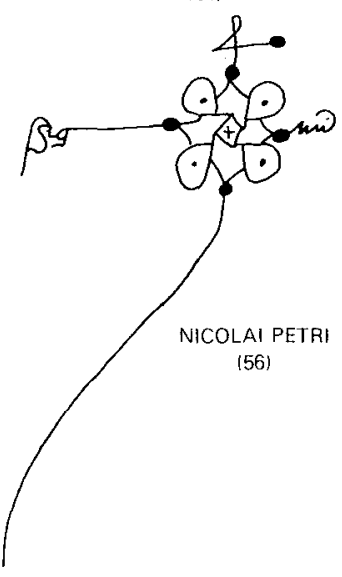

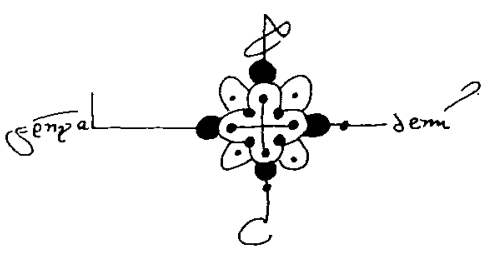

APPARICI GONCCALVEZ

(55)

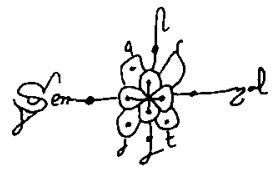

PERE CODINES

(57)

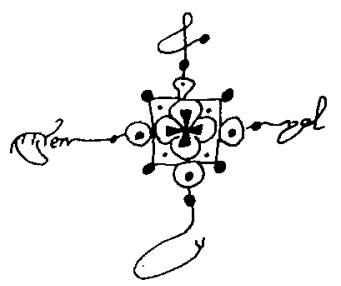

ANDREU FIRA

(58)

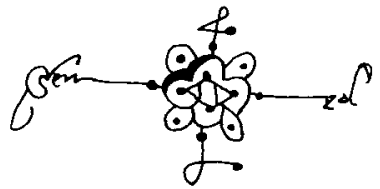

JOHAN DE MALLA

(59) 


\section{SIGLO XV}

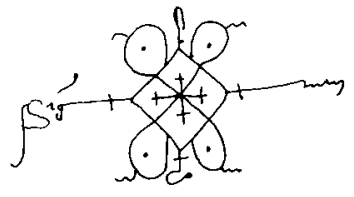

PETRUS GRANYANA (60)

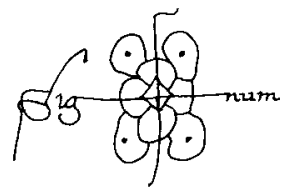

IOHANNES DE SANCTO FELICE (62)

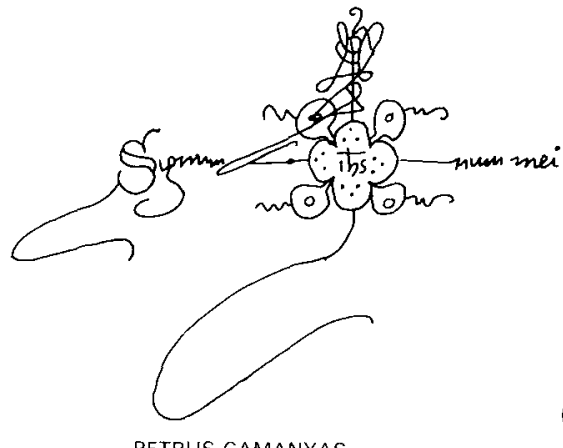

PETRUS CAMANYAS

(64)

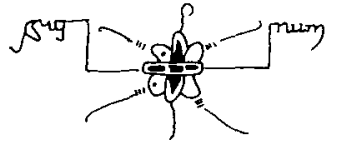

EDIGIUS PETRI DE BUYSAN (61)

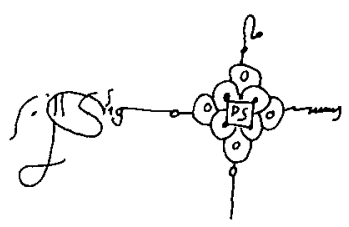

LUDOVICUS CARCASONA (63)

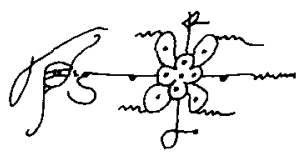

FRANCISCUS BATALLER (65) 